\title{
Les competences-socles : une nouvelle grille de lecture des constructions enfantines et de leurs anomalies
}

Hubert Montagner

\section{OpenEdition}

\section{Journals}

Édition électronique

URL : http://journals.openedition.org/communicationorganisation/1979

DOI : 10.4000/communicationorganisation. 1979

ISSN : 1775-3546

Éditeur

Presses universitaires de Bordeaux

\section{Édition imprimée}

Date de publication : 1 novembre 1997

ISSN : 1168-5549

Référence électronique

Hubert Montagner, «Les competences-socles : une nouvelle grille de lecture des constructions enfantines et de leurs anomalies », Communication et organisation [En ligne], 12 | 1997, mis en ligne le 26 mars 2012, consulté le 01 mai 2019. URL : http://journals.openedition.org/

communicationorganisation/1979; DOI : 10.4000/communicationorganisation.1979

Ce document a été généré automatiquement le 1 mai 2019.

(c) Presses universitaires de Bordeaux 


\title{
Les competences-socles : une nouvelle grille de lecture des constructions enfantines et de leurs anomalies
}

\author{
Hubert Montagner
}

1 En développant le concept de compétence pour désigner les capacités du bébé à percevoir les stimulations et informations de son environnement, en particulier celles qui émanent de sa mère, Brazelton a ouvert un champ d'investigation extrêmement fertile non seulement pour l'étude de la sensorialité et des capacités perceptives de l'enfant, mais aussi pour celle des ajustements qui fondent son attachement à sa mère, et réciproquement (1973, 1975, 1981, 1982, 1992). En effet, les compétences sensorielles et perceptives du bébé lui permettent à tout moment d'induire chez sa mère des comportements, actions, vocalisations et productions langagières (par induire, on entend « conduire ", « mener à une action, un comportement »: Larousse, 1983), de s'ajuster à « son rythme » (voir plus loin) et de l'entraîner à « son rythme » (Stern, 1982). C'est ainsi qu'un accordage ("attunement», Stern, 1985) peut s'établir et se développer entre l'enfant et sa mère, c'est-à-dire un ajustement de leurs émotions, et pas seulement de leurs comportements.

2 Les recherches que mes collaborateurs et moi-même avons effectuées depuis le début des années 70 à Besançon puis à Montpellier, et qui se poursuivent à Marseille, montrent plus généralement que le bébé et le jeune enfant ne sont pas des receveurs ou "réceptables " passifs qui répondent de façon univoque à telle ou telle stimulation ou sollicitation de leur partenaire du moment, leur réponse constituant elle-même une stimulation ou sollicitation qui modifie l'émetteur de façon prévisible. A tout moment explorateurs, «sélectionneurs ", décideurs et inducteurs, ils sont des acteurs souvent imprévisibles dont les capacités à « utiliser » le contexte et l'environnement organisent et régulent les comportements et représentations de leur partenaire. Ils peuvent moduler leur comportement en fonction de l'identité et des manifestations de celui-ci en tenant compte du contexte. Ainsi se trouvent «mis à mal» les modèles cybernétiques, 
behavioristes ou éthologiques "stimulus-réponse ", "émetteur-récepteur ou receveur ", "boucles de rétroaction» ou autres qui ont souvent nourri, et nourrissent encore, la réflexion théorique et les études comparatives sur la communication.

En fait, dès la naissance (et probablement la vie fotale), le bébé est capable de :

4 * choisir de répondre ou de ne pas répondre ;

$5 *$ modifier son comportement et son environnement, en conduisant ses partenaires à changer leur comportement et, s'agissant d'humains, leurs représentations et leur discours ;

$6{ }^{*}$ installer et réorienter une dynamique interactive dans laquelle «il pose les questions " et « apporte des réponses à ses questions", tout en répondant à celles des autres et en se « ménageant » des moments de non-réponse.

7 L'enfant humain apparaît ainsi comme acteur de son développement, tout en évoluant dans l'interdépendance de ses relations avec la ou les personnes d'attachement (Montagner, 1988, 1993,1996).

8 J'ai proposé le concept de compétence-socle pour rendre compte des capacités du bébé et $\mathrm{du}$ jeune enfant à conduire leurs interactions et processus de communication selon leurs particularités et celles de leur(s) partenaire(s), et plus généralement selon les particularités de l'environnement dans ses dimensions de relation, de temps et d'espace (Montagner, 1993, 1995, 1996). Cinq compétences-socles ressortent des observations « naturalistes » ou éthologiques et des expérimentations, mais aussi des études, pratiques et constats cliniques :

9 * la capacité d'attention visuelle soutenue, c'est-à-dire celle qui permet aux bébés, et plus généralement aux enfants de tout âge, de maintenir le regard focalisé sur une "cible», et de recueillir ainsi sans interruption des informations visuelles et auditives combinées (et aussi, dans les interactions proximales, les informations somesthésiques, proprioceptives, olfactives et gustatives qui leur sont associées) ;

10 * la capacité de manifester un élan à l'interaction, c'est-à-dire des comportements qui réduisent la distance, induisent la proximité et/ou le contact, puis l'interaction avec le partenaire (extensions du bras et de la main, caresses, sourires, mimiques, pédalages, bruits de bouche, cris, vocalisations, défécations, etc.);

$11^{*}$ la capacité à privilégier les comportements affiliatifs, c'est-à-dire ceux qui permettent de transmettre au partenaire une intention d'interaction sociale sécurisante, ajustée et accordée, et de recevoir les conduites de celui-ci sans insécurité, "malajustement » ou "mal-accordage », " désajustement » ou "désaccordage ", puis de poursuivre une interaction dans laquelle chacun paraît manifester une adhésion aux comportements et conduites de l'autre (fouissement, sourire et rire, caresse et prise de la main, vocalisation, offrande, sollicitation, doigt pointé, etc. ; paroles et discours chez les plus âgés). Il s'agit des manifestations qui nourrissent les processus dits de «socialisation";

12 * la capacité à organiser le geste de façon structurée et ciblée en direction des objets et des personnes ;

13 * la capacité à reproduire et imiter les actes, vocalisations, activités, paroles et conduites des autres (en d'autres termes, les processus d'imitation).

14 Ces cinq compétences-socles sont toutes « traversées » et « baignées » par le langage (qui ne peut pas ne pas être chez l'Homme). Elles sont aussi, et bien évidemment, «sous- 
tendues» par la nature même du fonctionnement neuronal qui rend absurde toute distinction fondée sur ce qu'on désigne par cognitif par rapport à ce qui ne le serait pas. En effet, chaque neurone intervient d'une façon ou d'une autre dans le décryptage du monde extérieur, et se trouve influencé à tout moment par son propre fonctionnement et celui des autres cellules nerveuses. Il est donc impliqué dans l'élaboration de la signification et du sens de toute information, même quand il s'agit d'une émotion. On ne voit pas quel mécanisme ou processus pourrait échapper au « domaine cognitif » (voir la discussion).

Les compétences-socles apparaissent indispensables aux constructions émotionnelles, affectives, relationnelles, sociales, « cognitives » et symboliques de l'enfant. Il y a en effet coïncidence entre leur non émergence ou leur non fonctionnalité, et l'apparition ou le renforcement de différentes difficultés ou anomalies du développement, du comportement et du psychisme.

16 Les compétences-socles constituent à tous les âges la trame obligée des processus de communication, tant pour les induire et les modifier que pour les éviter. On peut en donner des illustrations à travers quelques exemples. Nous les avons choisis dans deux domaines : ${ }^{*}$ la communication entre le bébé et sa mère $;{ }^{*}$ la communication entre jeunes enfants.

\section{La communication entre le bebe et sa mere.}

\section{Quelques expérimentations pionnières sur le face à face et le contact œil à œil dans l'interaction et la communication.}

17 Nombreuses sont les études expérimentales qui montrent l'importance des interactions face à face et œil à œil entre le bébé et sa mère dans les constructions enfantines où se trouvent mêlés émotions, affects, interactions sociales et processus dits « cognitifs », et à travers lesquelles se fonde et se consolide l'attachement entre les deux personnes. Spitz (1945, 1946, 1968 ; voir aussi Spitz et Wolff, 1946) observe ainsi que la présentation d'un visage de face a une forte probabilité d'induire le sourire chez un bébé, même lorsqu'il s'agit d'un masque, alors que le sourire se fige lorsque le visage est présenté de profil (le sourire est, selon lui, l'un des 3 organisateurs du psychisme de l'enfant). Dans leurs études effectuées avec des bébés âgés de quelques jours, Meltzoff et Moore $(1977,1992)$ montrent que le bébé développe une protrusion de la langue en réponse à une personne qui tire la langue. Tronick et al. $(1977,1978,1980,1984)$ analysent de façon détaillée comment, en situation de face à face, les expressions faciales d'un adulte, notamment le passage d'un visage souriant à un visage figé («still face»), modifient le comportement d'un bébé («expressions faciales», orientations du regard, agitations des bras et des jambes, productions vocales).

\section{Nos études : les compétences socles du bébé et du jeune enfant, fondements du développement émotionnel, affectif, relationnel, social et « cognitif ».}

18 Nous avons choisi d'illustrer le concept de compétence-socle, et les développements qu'il autorise, à travers deux exemples issus des études longitudinales de l'un de nos groupes 
de recherche : * le premier concerne un bébé «qui va bien » et une mère en difficulté psychologique au cours de sa grossesse ; ${ }^{*}$ le deuxième se rapporte à un bébé chez lequel le diagnostic d'autisme a été posé à l'âge de 5 mois et une mère sans apparente difficulté majeure aux plans somatique et psychique. Les dyades mère-enfant sont d'abord suivies à la Maternité 3 ou 4 jours après l'accouchement, puis au domicile familial à des intervalles et fréquences aussi réguliers que possible (c'est-à-dire, le plus souvent, lorsque l'enfant est âgé de 1, 4, 8, 12, 18 et 24 mois, et parfois 6 et 10 mois). L'enfant et sa mère sont filmés sans interruption pendant lh30 à 3 heures : les prises de vues commencent pendant le premier épisode de sommeil diurne de l'enfant, c'est-à-dire entre 9 et 10 heures (pour un premier éveil « diurne » et un premier repas vers 6 heures), et se poursuivent de 30 à 60 minutes après le repas et les soins corporels qui sont donnés après l'éveil «spontané ». L'enfant est aussi filmé dès l'âge d'un mois au cours de ses interactions avec les autres personnes du milieu familial (père, frère, sœur, grand-mère, ami, chercheur, etc.), le plus souvent pendant la " phase » qui suit le repas. Nous pouvons ainsi procéder à une étude qualitative et quantitative de ses comportements et interactions au moment de l'éveil, dans l'attente de l'alimentation au sein ou au biberon, pendant le repas et les soins corporels, et dans les diverses situations post-alimentaires dont certaines sont prévues par le protocole (les autres sont créées à l'initiative des personnes du milieu familial : présentations d'objets, activités ludiques, etc.). Les films sont analysés au 1/10e de seconde.

\section{Les interactions et la communication entre un bébé « qui va bien » et une mère en difficulté psychologique pendant sa grossesse.}

19 Séparée de sa famille, la mère apparaît en difficulté psychologique lors de sa rencontre avec la pédopsychiatre au moment de l'écographie du 7e mois, qui révèle un retard de croissance du foetus : elle est dépressive et anorexique. Evoquant ses propres difficultés d'enfant avec sa mère, elle n'a pas encore développé «l'idée d'être mère » et ne parle pas du bébé « à venir » (il s'agit d'une fille).

À 3 jours, il est demandé à la mère, selon le protocole, d'entrer en interaction et de communiquer avec son bébé de chaque côté du berceau (où il repose sur le dos), selon les modalités qu'elle a choisies (le bébé est filmé dans les mêmes conditions lorsqu'il se trouve en situation d'interaction avec un chercheur, et lorsqu'une série de stimulations sonores est émise selon un ordre aléatoire: Montagner, 1993, 1996 ; Montagner et al., 1989 ; Babassud, 1996 ; Pages, 1996).

21 Ce bébé a clairement la capacité d'accrocher le regard de sa mère et de se laisser accrocher le regard par elle (Fig. 1). En outre, il a la capacité de se laisser piloter le regard non seulement par celui de sa mère, mais aussi par celui du chercheur. Enfin, il a déjà une capacité d'attention visuelle soutenue vis-à-vis du regard et du visage de ses partenaires du moment (mère et chercheur), c'est-à-dire une capacité à maintenir le regard focalisé sur ces zones corporelles pendant au moins 3 secondes (en fait, entre 5 et 10 secondes chez cet enfant, la durée pouvant être beaucoup plus élevée chez d'autres enfants au même âge). Le bébé a ainsi un temps suffisant pour capter les messages émotionnels et affectifs "véhiculés" par les variations du regard de son partenaire, ainsi que par les combinaisons de regards, mimiques, postures de la tête, bruits de bouche, vocalisations, contacts, caresses, mouvements de la main et des doigts dans son champ visuel, productions langagières. Il peut «construire» ses processus de discrimination et de 
reconnaissance des différents visages et, de façon combinée, des voix, sécrétions (odeurs et saveurs), contacts, pressions corporelles (stimulations proprioceprives) et «rythmes d'action » (Montagner, 1996, 1997) qui leur sont associés. Il peut ainsi donner signification et sens non seulement aux manifestations qui lui sont adressées, mais aussi aux perceptions que développent ses partenaires vis-à-vis de ses propres manifestations.

Le développement concomitant d'une autre compétence-socle, présente elle aussi dès la naissance, l'élan à l'interaction (les comportements d'attachement proposés par Bowlby, 1958, 1960, 1969, 1973, 1980), permet au bébé de mobiliser en situation proximale sa capacité d'attention visuelle soutenue dans des conduites d'attention visuelle conjointe avec ses partenaires, c'est-à-dire centrée sur la même " cible ». Les conditions sont créées pour que d'autres compétences se révèlent et se développent, notamment dans la dynamique des processus de communication.

La figure 2 donne un exemple de quelques-unes de ces constructions chez le bébé de la figure 1, alors âgé d'un mois : extraites du film vidéo, les photographies sont séparées par des intervalles de temps réels, depuis le temps T 1 « 1 heure 33 minutes 8 secondes 8 centièmes » (photographie $n^{\circ} 1$ ) jusqu'au temps $T 13$ « 1 heure 40 minutes 20 secondes 91 centièmes (photographie $\mathrm{n}^{\circ} 13$ ).

Positionné en face à face par sa mère à la demande du chercheur, conformément au protocole, le bébé montre d'abord sa capacité d'attention visuelle soutenue en focalisant son regard sur la partie inférieure du visage maternel (1). Puis, il « redresse » la tête pour établir un contact œil à œil, dépassant ainsi l'immaturité tonico-posturale qui empêche habituellement un enfant d'un mois de maintenir un port de tête non oscillant (2). Il accentue en même temps l'orientation « ciblée » des bras et mains en direction du visage de sa mère. Une telle combinaison a une forte probabilité d'induire à tous les âges le rapprochement et le contact corporel : nous la considérons comme la manifestation d'un élan à l'interaction. La mère répond en accentuant son sourire (2).

Lorsque la mère soutient la tête du bébé à la demande du chercheur (comme cela est prévu par le protocole), l'enfant « libère » son regard du contact œil à œil, et l'oriente au dessus de la tête de sa mère, tout en laissant les bras « retomber » le long du corps (3). Puis, il accentue l'appui de sa tête sur la main de sa mère, donnant ainsi l'impression d'être capté par ce qu'il regarde et de transmettre un «signal » (4). La mère manifeste d'abord un élan à l'interaction: elle embrasse son bébé (5). Ce comportement pourrait être soit une réponse à celui que manifestait l'enfant quelques secondes auparavant, soit une tentative pour capter de nouveau son regard et induire chez lui un nouvel élan à l'interaction. En tout cas, il est induit par un « enfant inducteur ", lui-même « induit » par l'environnement.

26 La mère adopte ensuite une attitude de recul et d'observation (6) qui la conduit à orienter tout le corps du bébé en direction de la " cible " qu'il regarde, après l'avoir " découverte " elle-même (7). Elle se lève (elle était assise) et conduit l'enfant à proximité de l'objet regardé (8). En un peu plus de 3 secondes (3,14 secondes), le bébé déploie un bras et une main, et amène celle-ci au contact de la « cible » (un mobile de couleur bleue suspendu au plafond) (8 à 10). Le regard de la mère suit la main en mouvement (8 à 10) jusqu'au contact avec la «cible » (11), en même temps qu'un sourire éclaire «son regard et son visage ». Alors que les doigts du bébé se referment sur le mobile, la mère oriente le regard vers son visage, comme si elle y recherchait une expression reflétant son « exploit », ou s'attendait à l'émergence d'une nouvelle compétence (11). 

ici l'extension du bras et des doigts, à partir de laquelle se développent les actes de saisie des objets, les sollicitations manuelles, les offrandes, les actes de désignation avec le doigt pointé, etc (voir plus loin). La mère révèle, quant à elle, la surprise et le plaisir que lui a procuré l'observation de cette "émergence ». Elle crée quelques minutes plus tard une situation susceptible d'induire le même comportement en présentant un objet dans le champ visuel du bébé (12). Elle induit ainsi effectivement un transport coordonné de la main du bébé au contact de l'objet (13). socles que nous avons distinguées, même si celles-ci peuvent aussi être fonctionnelles plus tôt : la capacité de privilégier les comportements affiliatifs et la capacité de produire des comportements imitatifs. Mais, à l'âge d'un mois, ces compétences sont habituellement plus discrètes et moins clairement observables que les trois autres. La figure 3 en donne un exemple chez l'enfant des figures 1 et 2, mais à l'âge de 4 mois, dans le cadre d'une interaction avec une personne non familière, en l'occurrence l'un des chercheurs: le bébé sourit franchement (comportement affiliatif) et l'imite (il tire la langue en réponse au partenaire qui tire la langue). Tout en manifestant un élan marqué à l'interaction (il conserve la tête penchée vers l'avant et maintient ainsi une étroite proximité avec le visage du partenaire), il mobilise sa capacité d'attention visuelle soutenue vis-à-vis du regard de celui-ci (et non de sa zone orale).

Plus généralement, selon les situations et les partenaires, la plupart des enfants âgés de 4 mois présentent déjà une gamme diversifiée de comportements affiliatifs : outre des sourires fréquents et durables, ils peuvent manifester des comportements d'offrande (jouets, nourriture,...), des comportements de sollicitation manuelle (extensions du bras avec la main en pronation, latérale ou même en supination), des extensions du bras avec le doigt pointé, des vocalisations modulées et/ou des pédalages «jubilatoires » (voir plus loin). Dans la figure 3, c'est le chercheur qui, en raison du protocole, a pris l'initiative de tirer la langue au bébé après l'avoir pris à bout de bras, et qui a ainsi induit son sourire et sa protrusion de langue. Cependant, on peut faire l'hypothèse que, par la forme, la fréquence et la durée de ses sourires et expressions faciales, le bébé induit lui-même des modifications et ajustements chez son partenaire, non seulement dans son regard et ses expressions faciales (conformément au protocole, le chercheur ne parle pas), mais aussi dans les pressions corporelles qu'il exerce (en maintenant l'enfant à bout de bras avec plus ou moins de stabilité, le chercheur «sollicite» les systèmes somesthésiques, proprioceptifs et vestibulaires du bébé).

En développant ses $\mathbf{5}$ compérences-socles, et en les rendant fonctionnelles dans ses interactions et processus de communication avec ses différents partenaires, l'enfant peut donc induire à tout moment leurs réponses, puis les réorienter ou les modifier, selon sa décision et son projet. Parallèlement, il peut décider de se placer dans des situations où ses actes et vocalisations sont induits par son partenaire, ou de résister à ses manifestations. Il apparaît ainsi acteur, sélectionneur, explorateur et 
manipulateur dans ses initiatives et ses réponses. La découverte de ses compétences par sa mère, son père et ses autres partenaires, et les modifications qui en résultent dans leurs représentations et projections, nourrissent une flexibilité qui permet à l'enfant de réguler, au fil de son développement, ses émotions et affects, ses systèmes relationnels, ses processus " cognitifs", son activité intellectuelle et ses projets, tout en restant inséré dans ceux des autres.

Un bébé âgé de 5 mois chez lequel un diagnostic d'autisme a été posé par le neuropédiatre, et une mère sans difficulté majeure aux plans somatique et psychologique (aucune photographie n'est proposée pour des raisons éthiques)

C'est à la demande d'une pédopsychiatre de notre groupe de recherche que, conformément au protocole habituel (Montagner, 1993, Montagner et al., 1989; voir le précédent exemple), nous avons organisé à partir de l'âge de 5 mois et demi le suivi d'une petite fille à intervalles réguliers pendant près de 6 ans (nous ne disposons pas d'enregistrements filmés pour les premiers mois). Le motif donné par les parents au moment de leur demande de consultation est constitué par les difficultés d'alimentation de l'enfant. Mais, les médecins constatent aussi que la petite fille ne répond à aucune tentative de sollicitation par le regard, le toucher, le geste, les vocalisations, les paroles ou les bruits, quel que soit le partenaire. Elle ne répond pas davantage à la présentation d'objets ni à la production de bruits. Selon le neuro-pédiatre, la petite fille est autiste.

Nos observations sont en accord avec celles des cliniciens : quels que soient le partenaire, la situation et le contexte, le regard de l'enfant n'accroche aucune cible, et ne se laisse accrocher par aucun regard. La petite fille ne présente donc aucune interaction œil à œil. En outre, on n'observe chez elle ni élan à l'interaction, ni comportements affiliatifs, ni organisation structurée et ciblée du geste, ni comportements imitatifs. Son visage est inexpressif et ses actes de saisie des objets sont inexistants. Ses seules manifestations vocales sont des gémissements. Portée à bout de bras, elle a les bras et jambes ballants, sa tête bascule et s'affaisse. Placée sur le ventre, elle ne redresse pas la tête. Son corps s'affaisse et se tasse lorsqu'elle est installée dans un «baby relax ». Elle présente donc des déficits tonico-posturaux importants. Cette petite fille ne paraît avoir aucune possibilité d'induire l'interaction et la communication, pas plus que ses différents partenaires (mère, père, frère âgé de 3 ans, grand-mère, chercheur) ne parviennent à induire chez elle la moindre réponse.

Deux semaines plus tard (l'enfant a 6 mois), les observations sont inchangées pendant la première heure de la session expérimentale, jusqu'au moment où la mère décide de changer la couche de l'enfant. Le dos moulé et la tête soutenue par le matelas à langer, et ses déficits tonico-posturaux ainsi "compensés ", la petite fille révèle pour la première fois qu'elle a les capacités d'accrocher le regard de sa mère, et aussi le regard du chercheur, de se laisser accrocher et piloter le regard, même si la durée du contact œil à œil est toujours brève ( 1 à 2 secondes). En outre, elle ébauche des sourires, agite les jambes et vocalise, conduisant ainsi sa mère à se rapprocher, sourire, vocaliser, caresser sa tête et parler. À son mari qui vient d'entrer dans l'appartement, elle dit «tu comprends, elle ne peut pas faire deux choses en même temps: tenir sa tête et communiquer ». Elle vient de prendre conscience de l'existence d'un lien fonctionnel entre les déficits tonico-posturaux de l'enfant et ses déficits à entrer dans le jeu des interactions et de la communication avec autrui. Cette « découverte » a rassuré la mère et modifié ses représentations. Rentrée de son travail (l'enfant est gardée par la grand-mère 
maternelle), elle recherche désormais activement les situations et contextes susceptibles d'induire les phénomènes observés au cours de la session expérimentale. Convaincue que sa fille a d'autres compétences cachées, elle ne la confine plus à son lit et à son baby relax. En l'installant sur une couverture posée à même le sol, elle lui donne la possibilité de révéler des capacités motrices et locomotrices inattendues. L'enfant a effectivement montré au fil de ses deuxième et troisième années qu'elle pouvait se retourner, puis ramper, se déplacer par quadrupédie, se dresser sur ses jambes, marcher et enfin escalader, même si la progression était en décalage par rapport à celle, plus précoce et "accélérée ", des enfants "ordinaires". En développant son autonomie locomotrice pendant ses $2^{\text {ème }}$ et $3^{\text {e }}$ années, l'enfant a rencontré des obstacles et des objets qui lui ont permis d'induire de nouveaux comportements et de nouvelles représentations chez sa mère et les autres personnes de la famille, en particulier son frère. C'est essentiellement par de nouvelles formes d'activité (par exemple, sortir des couverts d'un tiroir et les poser sur la table, escalader la barrière empêchant l'accès au salon...) et des " productions sonores » (cris, vocalisations, bruits d'un objet porté au contact d'un autre ou du sol) que la petite fille induit chez ses partenaires familiers le rapprochement et la recherche d'interactions. Entre 2 et 3 ans, elle accepte aussi progressivement l'accrochage de son regard par celui de son partenaire, même si la durée du contact œil à œil reste brève (inférieure à 5 secondes), et n'évite plus le regard d'autrui, quel que soit son positionnement corporel (assise ou debout sur le sol, dans les bras, etc.), et même si son visage reste inexpressif. À 4 ans, elle présente des sourires, même s'ils sont peu marqués, vocalise, offre des objets, sollicite avec la main et tente de reproduire certains gestes et certaines vocalisations et activités de ses partenaires, essentiellement de son frère. C'est aussi ce qu'on observe à 5 ans avec d'autres enfants lorsqu'elle est reçue à temps partiel dans une école maternelle. En outre, elle n'évite pas ses "pairs ", et même s'en approche, entre dans l'enchaînement de leurs poursuites ludiques, essaie de prendre leurs objets, entre en conflit avec eux, reçoit leurs offrandes et ébauche parfois des activités de coopération. Elle induit ainsi chez eux des attitudes, gestes, contacts, vocalisations et discours (qui lui sont adressés), ainsi que des réponses à ses manifestations. C'est en même temps ses modalités d'occupation de l'espace (courses, escalades...), ses choix et manipulation d'objets, ses présences et participations aux activités collectives, son « intégration» dans celles-ci (par exemple, des enfants la prennent par la main et l'entraînent dans une ronde, ce qui est facilité par les demandes des institutrices), qui induisent chez les "pairs» des débuts d'interaction et aussi des communications dans lesquelles ils "produisent» les questions et les réponses. Mais ses compétences-socles sont encore insuffisamment structurées, "lisibles » et fonctionnelles pour induire une communication élaborée. En effet, outre que son langage se limite à quelques mots, la petite fille ne développe pas une attention visuelle soutenue vis-à-vis du regard et du visage de ses partenaires (c'est seulement à une certaine distance qu'elle maintient le regard de façon relativement durable dans leur direction), son élan à l'interaction est diffus (il n'est pas clairement marqué et « ciblé » par rapport au partenaire) même si elle accepte le contact, ses comportements affiliatifs sont seulement amorcés ou ébauchés, ou ne sont pas clairement manifestés, et ses imitations se limitent à certains gestes, vocalisations et débuts d'activité.

Les décalages dans le développement de l'enfant ont été estimés trop importants, et ses possibilités ou capacités d'apprentissage trop limitées, pour qu'elle soit encore accueillie à l'école maternelle à 6 ans. Elle a été alors confiée à un hôpital de jour. 
Pourtant, cette petite fille a révélé qu'elle avait la capacité d'induire chez les autres des comportements, vocalisations et discours, et la possibilité d'avoir elle-même des comportements, vocalisations et paroles induits par ces manifestations. En outre, ses compétences-socles affleurent et peuvent être fonctionnelles, même si elles ne sont pas tout-à-fait structurées et "lisibles ", et même si leur " émergence " est incomplète et limitée seulement à certains moments et certaines situations ou certains contextes. En tout cas, elles ne sont pas inexistantes, et sont observables par les êtres d'attachement, c'est-à-dire la mère et le frère (le père a quitté le domicile familial), et par les "observateurs" expérimentés (l'institutrice et les chercheurs). On peut faire l'hypothèse que si ces compétences avaient été tout-à-fait bien observées et reconnues dès leur émergence par l'ensemble des partenaires du milieu familial (notamment la grand-mère qui gardait l'enfant en l'absence de la mère), puis nourries par une mère apaisée (son lieu de travail est éloigné de son domicile, sa situation professionnelle est précaire, son mari au chômage se sépare d'elle et ses relations avec sa propre mère, agressive et culpabilisante, sont à la fois conflictuelles et de forte dépendance), la petite fille aurait pu les développer avec ses différents partenaires familiaux, et être ainsi plus inductrice d'interactions et de processus ouvrant des voies à une communication plus élaborée. On peut aussi penser que si la mère avait pu la confier à une personne ou une institution qui puissent et sachent «lire" ses compétences émergentes, leur donner du sens et les rendre fonctionnelles, elles auraient été inductrices d'interactions mieux structurées et de voies pour la communication. C'est en tout cas ce que suggèrent les études que nous avons effectuées avec des enfants autistes et des enfants polyhandicapés (atteints d'infirmité motrice d'origine cérébrale), quel que soit «le poids» réel ou supposé des influences génétiques ou apparues pendant la vie prénatale ou autour de la naissance.

\section{La communication entre enfants}

Deux recherches nous ont permis de préciser les mécanismes et processus qui fondent les compétences-socles du jeune enfant, ainsi que leurs fonctions :

\section{Les interactions et la communication entre deux jeunes enfants âgés de 4 à 6 mois, assis dans des sièges dont la configuration " compense » l'immaturité tonico-posturale des enfants de cette classe d'âge.}

Grenier (1981) a été le premier à montrer que le soutien manuel de la tête d'un bébé de moins de 2 mois maintenu assis sur le bord d'une banquette (et dont l'abdomen est soutenu par l'autre main du partenaire), « libère » son regard et sa motricité. Partant de ce travail, nous avons conçu des sièges dont la configuration permette aux jeunes enfants qui y sont assis de «libérer » leurs capacités cachées, échappant alors aux contraintes posées par leur immaturité tonico-posturale, à un âge (4 à 6 mois) où ils peuvent cependant assurer une certaine régulation de leur port de tête et de leur équilibre corporel (Montagner, 1993 ; Montagner et al., 1990, 1993, 1994). Montés sur rails, deux de ces sièges peuvent accueillir deux enfants dont le niveau global de développement est le 
même, et dont les potentialités et capacités sont comparables, ou deux enfants qui présentent des différences développementales, comportementales ou autres.

La figure 4 illustre les 4 premières phases du protocole (chaque phase a une durée de 3 minutes) qui a permis de suivre les mêmes "paires " pendant 4 semaines consécutives à une semaine d'intervalle, les enfants de la même "paire» se rencontrant pour la première fois à l'occasion de la première session expérimentale (c'est aussi la première fois que les mères se rencontrent) : * les deux enfants sont en situation d'interaction avec leur mère au cours des deux premières phases. Dans la première (1), la mère a pour consigne d'établir et de développer la communication avec son enfant comme elle le fait habituellement. Dans la deuxième (2), il lui est demandé d'utiliser successivement trois objets dans ses interactions avec l'enfant, à raison d'une minute pour chacun; * la troisième phase met chaque enfant en situation d'interaction avec une étudiante inconnue, après que les mères se sont éloignées de 2 mètres environ, tout en restant dans le champ visuel des enfants ( 3$) ;^{*}$ dans la quatrième phase, les sièges sont orientés de $3 / 4$ l'un par rapport à l'autre, puis rapprochés jusqu'à une distance de 15 ans environ (4; voir aussi la photographie 1 de la figure 5).

Au cours de la cinquième phase, les sièges sont encore rapprochés jusqu'à ce qu'ils se touchent, mais conservent la même orientation l'un par rapport à l'autre (figure 5). Ils sont ensuite mis face à face (phase 6 : figures 5 et 6 ). Dans la septième phase, les dossiers sont inclinés vers l'avant, ce qui modifie le centre de gravité des enfants. Enfin, les dossiers redressés, les deux enfants reçoivent chacun un objet identique (phase 8).

Chaque "paire » est suivie le même jour de la semaine (mardi ou jeudi) et à la même heure (entre 10 et 11 heures) pendant les 4 sessions expérimentales (les conditions et le protocole restent inchangés).

\section{Premier exemple.}

42 La figure 5 montre comment, et dans quelle dynamique interactive, deux garçons de 4 mois et demi, a priori à des niveaux de développement comparables, révèlent et sollicitent mutuellement leurs compétences-socles, et peuvent développer une communication déjà complexe au cours des phases 4 (photographie 1), 5 (2 et 3) et 6 (4 à 6) :

\section{L'attention visuelle soutenue}

43 De la photographie 1 à la photographie 6 , c'est-à-dire pendant plus de 5 minutes (5 minutes 53 secondes 60 centièmes), les deux enfants maintiennent une attention visuelle soutenue l'un vis-à-vis de l'autre (en fait, pendant les 9 minutes des phases 4, 5 et 6):

* Pendant plus de 2 minutes, les enfants maintiennent leur regard accroché l'un à l'autre (photographies 1 et 2). Puis, les regards glissent en direction du pied «actif»du partenaire, d'abord chez H (3), puis chez I (4). L'attitude «initiale» d'observation soutenue des deux enfants permet à chacun d'ajuster à tout moment son comportement à celui de l'autre, en l'occurrence l'extension du bras suivie de la prise de la main de l'autre (2), puis la séparation des mains (3) et les « battements de pied » presque synchrones (3) ou en alternance (4 et 5$)$. 
C'est au moment où les deux enfants accrochent de nouveau le regard de l'autre qu'ils «transportent » une nouvelle fois le bras en extension dans sa direction, conduisant ainsi les doigts déployés à la rencontre de la main du partenaire (6).

\section{L'élan à l'interaction}

On observe en permanence des mouvements qui réduisent la distance entre les deux enfants, à l'exception du moment où I se cale sur son siège en position d'observateur (3) :

47 * $\mathrm{H}$ reste penché en avant pendant toute la durée des phases 4,5 et 6 . À l'exception du moment où les mains se séparent et alors que $\mathrm{H}$ commence à lever la jambe droite (3), I adopte lui aussi une position de rapprochement avec son partenaire (1 et 2), puis se penche de nouveau en avant (4), contribuant ainsi à la réduction de la distance avec $\mathrm{H}$.

* Les deux enfants déploient en même temps un bras en direction du partenaire (2 et 6). Ils se retrouvent ainsi main dans la main (2), puis à la recherche d'un nouveau contact manuel avec les doigts en extension (6). Ils manifestent donc clairement un élan à l'interaction l'un vis-à-vis de l'autre quel que soit leur positionnement corporel.

\section{Les comportements affiliatifs}

C'est l'enfant qui manifeste sans interruption son élan à l'interaction (H) qui présente l'un des comportements affiliatifs les plus « lisibles ", le sourire (2 et 3), en même temps qu'il développe l'un des comportements qui ont une forte probabilité d'induire à cet âge le sourire du partenaire, c'est-à-dire l'agitation organisée des jambes (3), même si, dans le présent exemple, le sourire est seulement ébauché chez I (5). Cependant, les deux enfants font tous deux glisser leurs pieds l'un sur l'autre (2,4 et 5), ainsi que leurs mains (2 et 3). Le transport du bras et de la main en extension (1) a la même forme que dans la sollicitation de l'attention du partenaire, ou de l'objet qu'il tient, chez les enfants plus âgés (Montagner, 1978, 1988, 1993, 1996). La prise de la main du partenaire (2) est aussi comparable à celle qu'on observe chez les plus âgés lorsqu'ils ont établi entre eux des relations d'attachement. Nombre de comportements affiliatifs sont donc déjà bien fonctionnels entre enfants âgés de moins de 5 mois (voir aussi la figure 6).

\section{L'organisation structurée et ciblée du geste}

Les gestes des deux enfants sont à tout moment structurés, ciblés et organisés en fonction de la réponse de l'autre. Les mouvements de pieds de chaque enfant sont eux aussi structurés et organisés, à la fois par rapport à son activité manuelle et par rapport aux mouvements et gestes du partenaire (2 à 6).

\section{Les comportements imitatifs ou en miroir}

51 Les deux enfants conservent l'un par rapport à l'autre le même positionnement corporel (quasiment en miroir), à l'exception du moment où I adopte une attitude d'attente et d'observation (3). Leurs positionnements et mouvements des bras et des mains «se ressemblent " au moment où I commence à reproduire de façon presque synchrone le battement vertical de l'un des pieds de $\mathrm{H}$ (3), et après plusieurs imitations (selon le même rythme) des battements du pied de H par I (6). Les enfants de 4 à 5 mois ont donc déjà la capacité de reproduire les comportements d'un partenaire du même âge.

Communication et organisation, 12 | 2012 
52 En résumé, chaque enfant utilise à tout moment ses compétences-socles pour induire les réponses du partenaire et se laisser induire par celui-ci, non pas selon un schéma du type "stimulus-réponse", mais dans le cadre d'une dynamique où chacun combine les manifestations du partenaire et ce qu'il a décidé de privilégier: chez I, le regard est maintenu sans interruption en direction du visage du partenaire (1 à 3), avant de se focaliser sur le pied « actif » de celui-ci (4 et 5), puis d'accrocher de nouveau son regard (6) ; chez H, le regard glisse plus tôt vers les pieds (3), avant d'accrocher une nouvelle fois celui de I.

\section{Deuxième exemple}

53 La figure 6 donne un deuxième exemple de dynamique interactive entre deux autres garçons également âgés de 4 mois et demi, mais en situation de face à face (phase 6).

\section{L'attention visuelle soutenue}

54 L'attention visuelle des deux enfants est constamment mobilisée en direction du partenaire: les regards sont ciblés soit sur l'une ou l'autre des zones corporelles de l'autre, soit sur l'objet attaché à son corps (l'extrémité de la sangle qui relie les parties avant et arrière du " gilet » par l'entrejambe) pendant toute la durée de la séquence de la figure 6 ( 1 minute 11 secondes 8 centièmes) (en fait, pendant les 3 minutes de la phase) :

55 * L'enfant $\mathrm{R}$ a pour cible de son regard la ou les mains de $\mathrm{S}$, ainsi que la sangle de son « gilet » (photographies 1 à 6), puis il l'oriente vers la zone orale de $S$ après que celui-ci lui a pris la main (7). Ayant "rencontré » le sourire de S, R déplace de nouveau son regard vers la sangle (8), puis en direction du visage de son partenaire lorsque celui-ci s'est également saisi de l'objet (13). Cette fois, les deux regards se rencontrent (13), puis $\mathrm{R}$ déplace une nouvelle fois le regard vers la sangle (14).

56 * Après avoir été orienté en direction du pied de $\mathrm{R}$ (photographie 1), le regard de $\mathrm{S}$ se focalise sur sa main dès qu'elle touche sa propre main (3). C'est après avoir saisi la main de son partenaire que l'enfant $S$ cherche à accrocher son regard (7). Mais, l'enfant $R$ ne poursuit pas le mouvement qui aurait conduit à un contact œil à œil. Il fait de nouveau glisser son regard vers la sangle, induisant ainsi l'orientation du regard de $\mathrm{S}$ en direction de la même cible (8). Les deux enfants se retrouvent ainsi dans une situation d'attention visuelle conjointe (9 à 11). C'est la reprise de la sangle par $\mathrm{R}$ qui induit de nouveau la réorientation du regard de l'enfant $\mathrm{S}$ vers le visage de son partenaire et aussi l'extension de la main avec le doigt pointé vers ses yeux (12).

\section{L'élan à l'interaction}

57 Les deux enfants manifestent à tout moment des comportements qui entraînent ou maintiennent une proximité corporelle l'un vis-à-vis de l'autre, même si elle est surtout induite par un intérêt commun pour le même objet. Cependant, l'enfant S montre aussi un élan à l'interaction remarquablement structuré et développé. Cette compétence se manifeste à travers différents comportements au cours de trois phases successives :

58 * détachant son regard de l'objet qui a retenu l'attention et l'intérêt de son partenaire R, l'enfant $\mathrm{S}$ se saisit de sa main (4 à 6) et la garde dans les siennes (7). 
59 * il développe avec lui une attention visuelle conjointe vis-à-vis de ce même objet, puis, en alternance, la même manipulation de l'objet (9 à 11).

* il transporte le bras et la main en extension à proximité du visage de R (12), puis au contact de sa tête (14) après l'avoir « conduit » à un contact œil à œil (13).

\section{Les comportements affiliatifs}

61 Les deux enfants conduisent leurs mains au contact l'une de l'autre à la suite de mouvements alternés et ajustés (1 à 4). Mais, c'est l'enfant dont l'élan à l'interaction est clairement manifesté (S) qui révèle les comportements affiliatifs le plus diversifiés et complexes :

$62 *$ la « caresse » de la main de l'autre (4).

63 * la prise de cette main (5 et 6) puis sa conservation dans ses deux mains réunies (7), comme les adultes le font souvent avec un enfant (et aussi entre eux) dans les interactions apaisantes ou rassurantes.

64 * la combinaison de ce pattern avec le sourire et la recherche du contact œil à œil.

65 * le transport du bras et de la main en extension, avec l'index pointé sur les yeux à proximité immédiate du visage de l'autre (12). Ainsi se trouvent combinés deux patterns couramment observés dans les conduites de sollicitation des enfants plus âgés: la sollicitation avec la main en pronation, et le doigt pointé vers le partenaire.

66 * le toucher léger (caresse de la tête) et la sollicitation posturale (la tête est inclinée sur l'épaule) (14).

\section{L'organisation structurée et ciblée du geste}

67 Tous les gestes des enfants sont structurés, ciblés et organisés à la fois par rapport au « projet » de chacun et par rapport au comportement du partenaire :

* le bras en extension, l'enfant $\mathrm{R}$ transporte avec précision la main gauche au contact de l'objet-cible (la sangle) en effectuant une rotation de la main pour qu'elle soit en position d'effectuer un acte de saisie ( 1 à 4$)$ : la main est à plat et les doigts sont en extension lorsque l'objet est atteint (4). Cette coordination visuo-motrice repose sur une attention visuelle soutenue et non interrompue. Induits par ce geste, le mouvement du bras, la rotation de la main droite et l'extension des doigts de l'enfant $\mathrm{S}$ sont comparables. Même s'ils sont décalés, ils constituent quasiment une «image en miroir» de ceux de R, mais avec une différence quant à la cible puisqu'il ne s'agit pas de l'objet mais de la main du partenaire.

$69 *$ les deux enfants se montrent aussi capables de coordonner les gestes de leurs deux bras et mains : $\mathrm{S}$ organise ses gestes de façon ajustée pour saisir la main de son partenaire, puis "l'enfermer » dans ses propres mains (5 à 7); R organise lui aussi ses gestes de façon ajustée pour saisir la sangle puis la manipuler ( 9 et 10$)$; alors que $S$ retient la sangle au moyen de la main gauche lorsque $\mathrm{R}$ l'a de nouveau saisie, il projette le bras droit de façon synchrone à proximité du visage de son partenaire, tout prêt de ses yeux, tout en développant un acte de désignation avec le doigt pointé.

\section{Les comportements imitatifs}


71 Comme dans l'exemple précédent, les deux enfants utilisent à tout moment leurs compétences-socles pour induire les réponses de l'autre et se laisser induire par lui, non pas selon un schéma du type "stimulus-réponse ", mais dans le cadre d'une dynamique où chacun combine les manifestations du partenaire et ce qu'il a décidé de privilégier : chez $R$, la prise et la manipulation de la sangle (1 à 4, puis 8 à 14); chez $\mathrm{S}$, l'interaction avec le partenaire, soit par des contacts de la main ou la tête et des sollicitations manuelles et posturales $(7,12$ et 14$)$, soit par une intervention dans son activité (9 à 11).

Les figures 5 et 6 montrent ainsi comment deux enfants placés dans une situation qui libère à la fois leur regard et leurs gestes, investissent leurs compétences-socles dans des interactions ajustées. Chacun découvre et conforte la signification que revêtent ses manifestations comportementales à travers la manière dont le partenaire les comprend, qu'il s'agisse de ses initiatives ou de ses réponses. En intégrant des éléments « lisibles » de l'autre dans son "répertoire" (orientations ciblées du regard, coordinations oculomotrices, caresses et prises de la main, manipulations alternées du même objet, postures corporelles, vocalisations, etc.), chacun est en situation d'intégrer en même temps l'émotion et l'intention du partenaire, et donc le sens que celui-ci donne à ses différents éléments de comportement. En combinant la signification qu'ils donnent à leur comportement et la perception du sens que l'autre lui attribue, les deux enfants dépassent le niveau des interactions ajustées et "se construisent l'un l'autre » dans un même système de communication.

\section{Les interactions et la communication entre jeunes enfants au sein d'un petit groupe}

Nous avons pu étudier comment le jeune enfant construit et développe des interactions encore mieux structurées et des communications encore plus élaborées dès lors qu'il peut évoluer en activité libre au sein d'un petit groupe de pairs (composé de 5 ou 6 enfants dans nos recherches), dans un lieu qui autorise la « conquête » de la troisième dimension de l'espace, c'est-à-dire la profondeur et la hauteur (la sécurité affective est assurée par la présence de deux adultes familiers, qui sont des éducatrices de crèche ou de pouponnière médicale pour des enfants accueillis dans l'une ou l'autre de ces structures). Les principaux aménagements de ce lieu sont représentés dans la figure 7.

La figure 8 donne un exemple d'interactions que permet la conquête de la profondeur, en l'occurrence celle d'une paroi percée d'orifices et de hublots qui peut être traversée, et donner lieu à des échanges de tout ordre depuis le couloir qu'elle délimite et l'espace ouvert de marche et de course où se trouvent les deux adultes, et inversement (Montagner, 1993 ; Montagner et al., 1993, 1994).

Attiré par l'apparition de l'enfant M (13 mois) dans le hublot, avec lequel il a des liens d'attachement, l'enfant o (11 mois) s'en est rapproché par quadrupédie, puis s'est mis debout en prenant appui sur la paroi (il n'a pas encore acquis la marche). En situation de face à face, les deux enfants ont développé plusieurs séquences d'interactions multimodales au cours desquelles ils ont été initiateurs et répondeurs, simultanément ou en alternance : il s'agit de successions de ruptures des regards et de combinaisons des regards accrochés l'un à l'autre, sourires, rires, sollicitations posturales et manuelles, cris, vocalisations, contacts des mains... (la photographie 1 montre le positionnement des enfants après ces enchaînements de séquences, juste avant la fin des échanges). C'est $M$ 
qui rompt l'interaction avant de disparaître du champ visuel de $O$ (2). Lorsqu'il fait apparaitre son visage derrière une autre ouverture de la paroi, $\mathrm{M}$ oriente son regard en direction de $\mathrm{O}$ en même temps qu'il vocalise et crie. Il attire ainsi son attention et induit son orientation corporelle (3), puis son approche et une nouvelle séquence d'interactions multimodales (4). Cette fois, c'est l'enfant 0 qui rompt l'interaction et disparaît à son tour du champ visuel de son partenaire (5). En appui sur la paroi, il se met debout en position d'attente à l'extrémité du couloir, se préparant à rencontrer de nouveau son partenaire (6 et 7). Cet enfant de 11 mois montre ainsi qu'il a déjà développé des capacités intégrant la permanence de «l'objet » disparu de son champ visuel et, s'agissant d'un partenaire familier, la représentation de son "projet». Il apparaît donc capable d'anticiper le comportement de $\mathrm{M}$. Après la réapparition de celui-ci (8), $\mathrm{O}$ se met à genoux au moment où le premier redresse le tronc, adoptant ainsi un positionnement corporel qui facilite «l'alignement» des regards (9). C'est également ce que donnent à penser les enchaînements suivants :

76 * O se met debout en appui sur la paroi au moment où $\mathrm{M}$, toujours à genoux, redresse presque complètement le tronc: les regards sont alors dans des plans horizontaux rapprochés (10).

77 * O passe à la quadrupédie (12) comme l'a fait M quelques secondes auparavant tout en regardant dans sa direction (11). Les regards se retrouvent ensuite au même niveau (12).

78 Les deux enfants mobilisent toute la gamme de leurs compétences-socles d'un bout à l'autre de cette succession d'enchaînements :

$79{ }^{*}$ ils manifestent une capacité d'attention visuelle soutenue l'un vis-à-vis de l'autre : leur regard est en permanence orienté en direction du partenaire, ou dans la direction d'où il « doit » venir.

80 * l'élan à l'interaction de chacun est sans cesse activé, non seulement par le comportement de l'autre, mais aussi par la représentation qu'il construit du comportement de celui-ci lorsqu'il disparaît de son champ visuel. C'est particulièrement clair pour $O$ qui anticipe le comportement de $M$ en se postant à l'extrémité de la paroi, et en attendant que celui-ci débouche du couloir d'où il " doit " venir après la rupture de la deuxième phase d'interactions. C'est également le cas de $\mathrm{M}$ qui, après la rupture de la première phase d'interactions, se poste derrière l'ouverture de la paroi et s'oriente par rapport au hublot, là où il s'attend à observer 0 . Les deux enfants mobilisent ensuite en permanence un élan mutuel à l'interaction. Les enchaînements observés sont comparables à ceux du jeu de cache-cache. Ils reposent sur des constructions mentales nourries par la permanence de "l'objet " et la représentation du comportement du partenaire à partir des interactions précédentes (ils se fondent aussi probablement sur leurs précédentes expériences et sur la connaissance que chacun a de l'autre en raison de leurs liens d'attachement).

$811^{*}$ Leurs comportements affiliatifs sont diversifiés et organisés en séquences structurées dans lesquelles se combinent les accrochages et pilotages des regards, sourires, rires, contacts manuels non agressifs, sollicitations posturales, manuelles et vocales. Parallèlement, les enfants ne manifestent ni comportements autocentrés, ni comportements de crainte ou de fuite, ni agressions, ni « hyperactivité ».

* leurs gestes, leurs modes de locomotion et leur positionnement corporel sont maîtrisés et organisés en séquences structurées et fonctionnelles. 
* l'imitation est permanente, que les deux enfants soient engagés dans des interactions face à face $(1,3,4)$ ou dans des ajustements posturaux et locomoteurs (9 à 12).

84 dimension de la hauteur. En effet, lorsqu'ils « colonisent » des superstructures (platesformes, mezzanines, etc.) avec des pairs, ils investissent leurs compétences-socles dans des interactions et communications complexes, inductrices de processus "cognitifs " nouveaux et de conduites élaborées (coopérations, entraides, activités symboliques, utilisations d'objets), qui s'inscrivent dans la troisième dimension de l'espace (Montagner, 1993, 1996 ; Montagner et al., 1993,1994).

\section{Conclusions et discussion}

Les études sur les interactions entre le bébé et sa mère montrent de toute évidence que le petit de l'Homme n'est pas un être passif qui subit les stimulations de son environnement. Il n'est pas "simplement " un receveur ou un réceptacle des informations que sa mère (ou toute autre personne d'attachement) sélectionne, filtre, amplifie ou module. Il est aussi émetteur, sélectionneur, "filtreur ", amplificateur et " moduleur ». C'est à la fois à partir de ses perceptions et de ses décisions qu'il établit, rétablit, développe, rompt ou refuse l'interaction, qu'il s'ajuste au comportement de son partenaire, et qu'il s'accorde avec les émotions de celui-ci. Dès la naissance, en tout cas dès les premiers jours, il est capable de réguler la communication en même temps qu'il se construit dans la communication. C'est ce que montre l'étude fine des interactions qui se développent entre lui et sa mère et, plus généralement, toute autre personne d'attachement. Mais les processus qui nourrissent les interactions ajustées et accordées, et conduisent aux communications les plus élaborées, ne peuvent être complètement appréhendés que lorsque l'enfant peut « libérer » toute la gamme de ses compétences. C'est ce qu'on observe à l'âge de 4 mois au moins (probablement plus tôt : étude en projet) lorsque, «libéré » des contraintes développementales posées par l'immaturité tonico-posturale, il est en situation d'interaction avec un partenaire dont le niveau de développement et les compétences sont comparables, et qui n'a pas avec lui une histoire et un vécu partagés, susceptibles d'inhiber, d'empêcher, de freiner ou de détourner l'émergence ou la fonctionnalité de telle ou telle compétence.

Le concept de compétence-socle permet de mieux cerner que les seules analyses cliniques et les études microscopiques (lorsque celles-ci se limitent à la quantification des seuls items de comportement) comment se construisent et se régulent les interactions et la communication chez le jeune enfant, quel que soit son partenaire. En effet, ce concept prend en compte de façon combinée les mécanismes et processus qui fondent le développement de la motricité corporelle et manuelle, de la sensorialité et de la perception, de l'attention, des émotions et des affects, des représentations, des conduites relationnelles et sociales, des activités symboliques, et de la connaissance de la signification ou du sens des indicateurs et signes de l'environnement. Le développement de l'être humain étant à tout moment spécifiquement ancré dans le langage (le fonctionnement du cerveau humain est, par essence, organisé autour du langage et par le langage), nous ne le tenons pas 
pour une compétence, mais comme une évidence "constitutionnelle" (ou structurelle). Il imprègne et traverse les 5 compétences-socles que nous avons distinguées, en même temps que celles-ci rendent le langage opératoire dans l'interaction et la communication. Il en de même pour la pensée qui nourrit les niveaux les plus complexes et abstraits du fonctionnement cérébral, et dont l'exploration échappe encore pour une très large part à l'investigation scientifique (peut-on vraiment et clairement définir ce qu'on entend par processus cognitif ? Lorsqu'un bébé discrimine et reconnaît au moins deux odeurs corporelles de sa mère, celles du sein et du cou - Montagner, 1988 - s'agit-il d'un processus seulement ou principalement sensoriel, cognitif, émotionnel, affectif ou relationnel ?). La pensée est toujours sous-jacente aux 5 compétences-socles.

\section{La capacité d'attention visuelle soutenue.}

Le bébé, nous l'avons vu, recherche et trouve «spontanément » l'accrochage visuel avec sa mère, en même temps que, par ses manifestations (orientations de la tête, et donc du regard, bruits de bouche, vocalisations, pédalages, extensions et "crispations" des mains...), il induit chez elle la recherche, l'accrochage et aussi le pilotage de son regard. L'orientation ciblée de son regard est guidée et facilitée par sa capacité à discriminer et percevoir les stimulations spécifiques (propres à l'espèce) et familières (mouvements de la main et des doigts qui attirent l'attention, configuration du visage; expressions faciales, bruits, vocalisations, voix et odeurs; contacts avec certaines zones corporelles, modalités du portage...) (Montagner, 1988, 1993 ; Babassud, 1996 ; Pages, 1996 ; Babassud, Pages et Montagner, en préparation). Ces mécanismes initiaux de captage et d'accrochage du regard de la mère (au sens large du terme : mère biologique, mère adoptive, père...), et les réponses ajustées de celle-ci, nourrissent chez le bébé une attention visuelle déjà bien soutenue. Elle devient de plus en plus soutenue à mesure que la mère elle-même ancre sa relation avec l'enfant dans la capture et le pilotage de son regard, en combinaison avec tout un ensemble de manifestations affectives (sourires, mimiques, caresses, bruits de bouche, vocalisations, paroles...). L'attention visuelle soutenue apparait ainsi comme une compétence indispensable à la « lecture » des émotions et des affects que véhiculent le regard et le visage maternels. Elle donne au bébé un temps suffisant pour donner une signification ou un sens aux expressions faciales et à la tonalité du regard. Il peut ainsi les associer aux intonations de voix, gestes, contacts et pressions corporelles... En outre, l'attention visuelle soutenue que manifeste l'enfant vis-à-vis d'une cible autre que sa mère a valeur de signal pour celle-ci, surtout après une interaction œil à œil également soutenue (fig.2). Le bébé induit alors chez elle non seulement une orientation du regard en direction de la cible, mais aussi un comportement et un discours focalisés ou imprégnés par celle-ci. En induisant chez sa mère une attention visuelle conjointe, le bébé lui donne la possibilité de comprendre ses possibilités et compétences. Parallèlement, l'enfant peut, à partir des manifestations maternelles, donner signification et sens à « l'objet-cible » et au partenaire du moment (une autre personne, un animal familier...).

Les figures 5 et 6 montrent que, dans la situation expérimentale qui permet de compenser l'immaturité tonico-posturale tout en assurant la verticalité du tronc, deux enfants âgés tous deux de 4 à 5 mois ont la capacité de maintenir une attention visuelle soutenue l'un vis-à-vis de l'autre pendant plus d'une minute (en fait, il est fréquent qu'ils le fassent plus longtemps, parfois tout au long des 3 minutes de la phase expérimentale) alors que, 
pourtant, les mères sont présentes et visibles. Cette compétence-socle remplit plusieurs fonctions essentielles, indispensables au développement individuel :

* elle autorise l'émergence et la maîtrise de coordinations oculo-motrices structurées et fonctionnelles, tant par rapport à la main, au pied et au visage du partenaire (fig.5) que par rapport à l'objet qui induit une attention conjointe (fig.6) : l'observation attentive donne à chaque enfant le temps de recueillir en continu des informations sur la dynamique gestuelle du partenaire par rapport à la configuration de l'objet, et d'organiser ses propres gestes en fonction des informations recueillies ;

* elle conduit à des ajustements précis du comportement à celui du partenaire, tant au plan de la forme (main en contact avec celle de l'autre, prise de sa main, imitation des battements de pieds, prise d'un objet) qu'au plan de la dynamique temporelle ;

$91 *$ * elle permet de donner signification et sens aux gestes de l'autre et à ses propres gestes, à partir de la « lecture » de son regard et de ses expressions faciales, et aussi de leurs combinaisons avec les postures corporelles, bruits de bouche, vocalisations, battements de pieds, pédalages, contacts et pressions qu'entraîne la rencontre des mains. Chaque enfant peut ainsi pénétrer dans le monde des émotions et des représentations, non pas seulement de sa mère et des autres personnes familières, mais aussi de tout partenaire rencontré à tout moment. C'est aussi les représentations de la mère, spectatrice muette, qui peuvent être modifiées à l'issue des sessions expérimentales par l'attention et l'intérêt que montre l'enfant pour les personnes nouvelles (l'autre enfant et sa mère), qui se comportent comme si elles le "considéraient" comme un partenaire à part entière ayant des compétences fonctionnelles, même lorsqu'il est "hypotonique", handicapé sensoriel ou moteur, trisomique même lorsqu'il est né prématuré, hypotrophe même lorsqu'il suscite l'inquiétude ou l'angoisse parce qu'il a un «retard» de croissance, des difficultés alimentaires (l'enfant refuse l'alimentation ou vomit ), des « troubles » du sommeil, etc.

92 Lorsque l'attention visuelle du jeune enfant est faiblement développée (le regard balaie la cible regardée sans s'y arrêter; le regard se pose sur la cible, mais ne l'explore pas : se maintenant en "point fixe », il ne peut être piloté) ou non observée (l'enfant n'accroche pas le regard du partenaire, ou l'évite), il ne peut développer ni coordinations oculomotrices structurées, ni attention visuelle conjointe, ni ajustement comportemental et émotionnel au partenaire, ni constructions psychiques qui intègrent la perception des représentations des personnes à travers leur regard, leurs expressions faciales et les manifestations associées. Mais, cette compétence-socle peut être «seulement " enfouie ou masquée: elle peut "émerger» dès que certaines conditions sont réunies (modification dans les représentations et conduites de la mère, interactions avec de nouveaux partenaires qui peuvent s'ajuster et s'accorder avec l'enfant, etc.) (Montagner, 1993,1996).

\section{L'élan À l'interaction}

93 Le bébé manifeste à chaque instant des comportements qui réduisent la distance et établissent la proximité puis le contact avec sa mère ou toute autre personne (bruits de bouche, succions, agitations des bras, extensions et « crispations » des mains, pédalages, réorientations de la tête en direction du partenaire, fouissements, mimiques, protrusions de la langue, vocalisations, cris, pleurs, défécations...). Ces comportements sont souvent 
interprétés non seulement comme la traduction de phénomènes biologiques ou psychophysiologiques (faim, sommeil...), mais aussi comme la manifestation d'une demande d'interaction ou d'un élan à l'interaction. En tout cas, ils induisent chez le partenaire un élan à l'interaction. 11 s'agit de comportements d'attachement (Bowlby, $1958,1960,1969,1973,1980)$.

La présence rapprochée du partenaire crée une situation qui permet au bébé de mobiliser efficacement sa capacité d'attention visuelle soutenue et de bien "lire " les émotions et affects véhiculés par le visage regardé. La proximité corporelle permet en effet de "compenser» les déficits de la discrimination visuelle, encore en cours de construction. Elle crée les conditions pour que des interactions accordées puissent se développer entre les deux personnes (Stern, 1985), c'est-à-dire non seulement des ajustements de leurs comportements, mais aussi de leurs émotions. "Lecteur» d'émotions dont il a découvert et conforté la signification et le sens, le bébé est en mesure d'anticiper le comportement de son partenaire. Il peut alors prendre l'initiative d'induire ou de piloter les réponses de celui-ci dans le cours d'une interaction qu'il a initiée, et de "transmettre » à ce partenaire des éléments de son répertoire, surtout lorsqu'une familiarité s'est installée, comme cela est le cas dans notre étude pendant les $3 e$ et 4ème sessions expérimentales avec les bébés installés dans les sièges que nous avons conçus.

Lorsque l'élan à l'interaction de l'enfant est faiblement développé (non lisibilité, faible fréquence et/ou non fonctionnalité des comportements qui induisent l'approche, la proximité et le contact) ou non observé (enfant dit passif, autocentré ou évitant), il ne peut induire des interactions ajustées avec ses partenaires. Il lui manque à tout moment les repères visuels, et aussi ceux les repères associés (notamment auditifs), qui sont non seulement indispensables au développement des capacités de discrimination des visages, des objets mais aussi à celui des constructions qui permettent de donner signification et sens à l'environnement, y compris aux émotions et représentations des différents partenaires. Mais, comme l'attention visuelle soutenue, l'élan à l'interaction peut être "simplement» enfoui ou masqué. Cette compétence-socle peut "émerger » dès que certaines conditions sont réunies (Montagner, 1993, 1996).

\section{La prédominance des comportements affiliatifs}

Nombre des manifestations du bébé qui indiquent ou créent un élan à l'interaction sont aussi interprétées par la mère ou toute autre personne comme des comportements affiliatifs, c'est-à-dire des indicateurs ou signes d'une adhésion à leurs comportements, émotions, affects, représentations et intentions, et aussi à leur discours (" tu m'appelles », " tu veux un baiser ", «tu me souris », « tu es d'accord », « tu sais que je suis triste», « tu me comprends", «tu me dis que tu es content», «je savais bien que tu voulais ta peluche ", etc.).

97 Ces manifestations « initiales » conduisent tout au long de la petite enfance à d'autres comportements affiliatifs, plus complexes et encore plus porteurs d'interactions sociales. C'est ce que montrent notamment les recherches que nous avons effectuées avec des enfants du même âge qui peuvent «libérer » leur regard, leurs gestes et l'ensemble de leurs actes et vocalisations (voir précédemment). On peut citer :

* les rires et jubilations ; 
99 * les sollicitations de l'attention ou des objets avec la main en pronation, dans un plan vertical ou en positionnement plus ou moins oblique; les actes d'abandon, chutes provoquées, bruits rythmés ou jets d'objets pour initier ou prolonger une interaction avec rires et jubilations (même s'ils sont parfois combinés à des cris et des pleurs); les offrandes et trocs;

$100^{*}$ les sollicitations très ritualisées et symboliques: les présentations de la main en supination, qui autorisent aussi la diversification des conduites d'assemblage des objets (emboîtements, encastrements, etc.) ; l'inclinaison latérale de la tête avec recherche du contact œil à œil. Elles apparaissent déjà bien développées entre 4 et 5 mois dans notre étude avec les enfants installés sur les sièges expérimentaux ;

$101 *$ * le doigt pointé en direction du partenaire au moment des rencontres et au cours des interactions, qui sont aussi des gestes de désignation des objets, des personnes et des lieux. Ce pattern est également déjà structuré et fonctionnel entre 4 et 5 mois ;

$102 *$ les caresses, enlacements et baisers;

103 * la mise en commun de comportements dans la réalisation d'une activité commune, c'est-à-dire les activités de coopération (les transports coordonnés des objets lourds ou encombrants d'un lieu à un autre, les constructions, etc.). Les coopérations entre enfants sont déjà élaborées et diversifiées dès le milieu de la deuxième année (Montagner, 1993, 1996 ; Montagner et al, 1993, 1994);

104 * l'entraide, déjà observée au cours de la deuxième moitié de la deuxième année.

105 L'ensemble de ces comportements affiliatifs constituent des socles essentiels des processus et conduites de communication les plus élaborés, dans lesquels l'induction réciproque est la règle et le moteur, en particulier :

$106 *$ les processus dits de socialisation, qui règlent et modulent les interactions sociales dans les groupes, et en organisent les dimensions les plus complexes dès la deuxième année ;

107 * les jeux de rôles et les activités symboliques, déjà développés entre 2 et 3 ans (Montagner, 1993, 1996 ; Montagner et al, 1993, 1994).

108 * la transmission aux autres de savoirs, langages, cultures et produits de la pensée, et en même temps «l'appropriation» de leurs savoirs, langages, cultures et produits de la pensée.

109 Lorsque les comportements affiliatifs sont faiblement développés, c'est-à-dire seulement ébauchés ou insuffisamment structurés, rares, non lisibles et/ou non fonctionnels (par exemple, lorsqu'ils sont "parasités" par des actes, vocalisations et productions langagières étranges), ou non observés (par exemple, dans certaines formes de l'autisme), les enfants ne peuvent disposer de fondements ou socles indispensables aux constructions les plus complexes et « auto-régulatrices » de la vie émotionnelle, affective, relationnelle, sociale, symbolique et intellectuelle. Le « handicap » est d'autant plus lourd que le faible développement ou la non émergence (ou non observation) des comportements affiliatifs sont combinés à une «expression» prédominante des conduites autocentrées, de crainte ou de fuite, «d'hyperactivité » et/ou "d'agression ». Cependant, les «balances comportementales» évoluent «au bénéfice» des comportements affiliatifs dès que les enfants peuvent évoluer dans des conditions qui les induisent et leur donnent signification et sens, permettant ainsi des modifications dans les représentations de chacun. Leurs interactions et processus de communication peuvent alors s'inscrire dans l'induction réciproque. Ils sont en mesure d'échapper à l'image et au 
statut qu'a nourri le faible développement de leurs comportements affiliatifs au cours des premiers mois ou premières années (enfants dits repliés ou murés sur eux-mêmes, voire autistes, insécurisés, craintifs et/ou fuyants, hyperactifs, agressifs et casseurs, etc.).

\section{L'organisation structurée et ciblée du geste}

110 Nous avons vu que, dès la fin du premier mois (encore plus tôt, selon d'autres études), le bébé a déjà la capacité de structurer et d'organiser son geste de façon précise vis-à-vis d'une cible qui a retenu son attention visuelle. Ce pattern est un socle obligé de l'ensemble des comportements de saisie et de préhension des objets, depuis les premiers temps du développement post-natal au cours desquels ceux-ci sont présentés dans le champ visuel du bébé jusqu'aux âges où l'enfant peut aller à leur «rencontre » et les explorer à son initiative (dès 4 mois au moins, comme le montre notre étude avec les enfants installés dans les sièges expérimentaux), puis les rechercher dans son environnement dès que son autonomie locomotrice l'y autorise. L'organisation structurée et ciblée du geste est évidemment indispensable pour qu'un enfant puisse modifier les objets, et notamment en créer de nouveaux en déformant et/ou assemblant plusieurs d'entre eux. Elle permet enfin à chacun d'explorer le visage du partenaire avec des gestes mesurés et ajustés qui sont interprétés comme des actes de tendresse et d'attachement. Elle a donc aussi des fonctions émotionnelles, affectives, relationnelles et sociales.

111 Comme précédemment, le faible développement (faible fréquence, non lisibilité et/ou non fonctionnalité), les formes étranges ou la non émergence (ou non observation) de cette compétence, s'accompagnent de difficultés dans les différentes constructions enfantines. Par exemple, lorsque le geste de projection frontale du bras et de la main en pronation, qui conduit à la fermeture des doigts sur un objet, n'a pas encore été manifesté (ou observé) à la fin de la première année, alors que la présentation de l'objet induit un mouvement enveloppant de l'un des bras ou des deux, et seulement un mouvement enveloppant. Ou encore, à 3 ou 4 ans, lorsque la saisie des objets reste lâche et ne permet pas de les retenir au moment où ils tombent, et lorsque les doigts ne se referment pas « en pince " pour tenir les « outils » du gribouillage, de «l'écriture », du dessin ou de la peinture. Cependant, l'organisation structurée et ciblée du geste peut, elle aussi, se développer dès que les interactions sont structurantes et organisatrices.

\section{La capacité de reproduire et d'imiter}

112 Le bébé a aussi la capacité de reproduire et d'imiter ses partenaires. Il répond dès les premiers jours par un gonflement puis une protrusion de la langue à une personne qui tire la langue. À cours du premier mois, il peut reproduire, au moins partiellement, des expressions faciales, des bruits de bouche et des vocalisations. Ses capacités de reproduction se diversifient dès lors qu'il peut se libérer des contraintes posées par son immaturité tonico-posturale, c'est-à-dire lorsqu'il peut assurer un équilibre corporel stable avec le tronc verticalisé. Il apparaît alors capable d'intégrer à son répertoire des patterns gestuels et vocaux de son partenaire, tant au plan de leur configuration que de leur rythme, puisqu'il peut les reproduire, tout en conservant ses patterns et enchaînements de patterns. Il peut en même temps adopter des positionnements corporels et gestuels « en miroir ». Il manifeste des capacités tellement développées de s'ajuster aux manifestations du partenaire qui traduisent un état de bien-être, de sécurité 
affective ou de non insécurité (sourires, contacts « de surface » main sur main et prises enveloppantes de la main qui conduisent à la main dans la main : figures 5 et 6 ; contacts, frottements et battements des pieds : figure 5), qu'il ne peut pas ne pas capter, capturer, percevoir et intégrer la signification et le sens des états intérieurs sous-jacents. Les interactions développées avec sa mère et les autres personnes familières depuis la naissance (et avant pour certains aspects) ont évidemment préparé cet "accordage " (Stern, 1985). À 4 mois et demi au moins, les enfants sont désormais capables non seulement de reproduire la forme et le rythme d'un comportement, mais aussi de traduire l'état intérieur qu'il reflète. Il est imitateur.

Le faible développement (faible fréquence, non lisibilité et/ou non fonctionnalité), les formes étranges ou la non émergence (ou non observation) de la capacité de reproduire et d'imiter les comportements, activités et productions langagières des partenaires, reflètent ou traduisent des difficultés émotionnelles, affectives, relationnelles, sociales et «cognitives» de l'enfant. En effet, il ne peut alors intégrer à son répertoire des manifestations parfois vues et entendues, mais qu'il n'a pas expérimentées, et dont il ne peut ainsi découvrir ou vérifier la ou les fonctions, la signification et le sens, et auxquelles il puisse, en les exécutant, donner d'autres fonctions, significations et sens à partir de ses propres expériences et acquisitions. Parallèlement, cet enfant a des difficultés à induire l'imitation chez ses partenaires. Mais, comme pour les autres compétences-socles, la capacité à reproduire et imiter peut se développer dès que certaines conditions sont réunies (Montagner, 1993, 1996).

114 Imprégnées et traversées par le langage, interdépendantes et interactives, les compétences-socles que nous avons distinguées fournissent donc une grille de lecture renouvelée qui facilite l'exploration des processus d'interaction et de communication entre l'enfant et ses différents partenaires :

115 * l'attention visuelle soutenue apparaît dès la naissance comme un socle obligé non seulement de toute prise d'informations identifiables par des caractéristiques physiques (forme, dimension, couleur, cinétique, etc.), mais aussi de la construction par l'enfant de leur signification émotionnelle, affective, relationnelle et sociale à partir de la «lecture " du regard et des expressions faciales de son ou ses partenaires, combinée au bain de langage, de vocalisations, de bruits, de contacts corporels, de sollicitations proprioceptives, d'odeurs et de saveurs où il est immergé. Ainsi peuvent s'installer les ajustements comportementaux et émotionnels qui fondent les interactions accordées dont se nourrissent les processus d'attachement.

116 * l'élan à l'interaction induit à tout moment l'approche, la proximité et le contact qui rendent opératoire l'attention visuelle soutenue en permettant au bébé d'identifier la présence sécurisante de sa mère et de ses autres partenaires familiers, et de mobiliser efficacement des capacités perceptives dont la dimension sensorielle n'est pas encore tout-à-fait construite.

117 * l'enfant est ainsi en mesure de montrer toute une gamme de comportements que les partenaires interprètent comme autant de manifestations d'adhésion (les comportements affiliatifs), et qui induisent chez eux des conduites affiliatives, inductrices elles-mêmes d'ajustements et d'accordages, et aussi de comportements nouveaux, inattendus et surprenants chez le bébé. Reconnu comme partenaire actif « qui a son mot à dire », celuici se construit comme un acteur qui donne du sens et du rythme à des échanges qui sont déjà des processus de communication. 
118 * les gestes peuvent alors se structurer et s'organiser vis-à-vis de toutes les « cibles ». Ils permettent à l'enfant d'intégrer les propriétés des objets à travers le regard, le discours et les conduites des partenaires. Il participe lui-même à l'induction de l'interaction et de la communication, en même temps qu'il est induit par elles.

119 * l'imitation permet au bébé de montrer à ses partenaires qu'il a intégré des éléments comportementaux et émotionnels de leur répertoire. En reproduisant eux-mêmes des manifestations de l'enfant, ses partenaires lui montrent qu'ils s'approprient eux aussi son registre. Au fil du développement, l'enfant peut ainsi mettre à l'épreuve ses capacités d'induction au cours de ses différentes rencontres, quels que soient les interlocuteurs et les lieux.

120 Combinées à l'attention visuelle (et auditive), à l'élan à l'interaction, aux comportements affiliatifs, à l'organisation structurée et ciblée du geste, l'imitation est aussi autoinductrice : elle conduit l'enfant à produire de nouveaux comportements et de nouvelles conduites, susceptibles d'induire l'imitation, elle-même inductrice d'interactions et de communications.

\section{BIBLIOGRAPHIE}

BABASSUD, M. 1996 «Etude comparative des compétences perceptives et interactives de bébés nés par césarienne et de bébés nés par voie naturelle ». Thèse de Doctorat en Sciences de la Vie et de la Santé, Université de Montpellier I.

BOWLBY, J. 1958 " The nature of the child's tie to his mother », Int. J. Psychoanal, 39, 350-373.

BOWLBY, J. 1960 « Separation anxiety », Int. J. Psychoanal., 41, 89-113.

BOWLBY, J. 1969 Attachment and loss. I: Attachment, London, The Hogarth Press and Institute of Psychoanalysis.

BOWLBY, J. 1973 Attachment and loss. II: Separation, anxiety and anger, London, Tavistock.

BOWLBY, J. 1980 Attachment and loss. III: Loss, sadness and depression, New York, Basic Books.

BRAZELTON, T.B. 1973 « Neonatal behavioral assessment scale », Clinics in Developmental Medicine, $\mathrm{n}^{\circ}$ 50, London, William Heinemann Medical Books; Philadelphia, Lippincott.

BRAZELTON, T.B. 1975 Early mother-infant reciprocity in parent-infant interaction, Amsterdam, Elsevier.

BRAZELTON, T.B. 1981 « Comportement et compétence du nouveau-né », Psychiatrie de l'Enfant, 24, 375-395.

BRAZELTON, T.B. 1982 « Joint regulation of neonate-parent behavior », in E.Z.Tronick (ed.), Social interchange in infancy, 7-22, Baltimore, University Park Press.

BRAZELTON, T.B. 1992 Touchpoints, New York, Addison-Wesley.

GRENIER, A, 1981 La « motricité libérée par fixation manuelle de la nuque au cours des premiéres années de vie », Arch. Fr. Pédiatrie, 38, 557-561. 
MELTZOFF, A.N., MOORE, M.K. 1977 « Imitation of facial and manual gestures by human neonates ». Science, 198, 75-78.

MELTZOFF, A.N., MOORE, M.K. 1992 « Early imitation within a functional framework: The importance of person identity, movement, and development ", Infant Behavior and Development, $15,479-505$.

MONTAGNER, H. 1978 L'enfant et la communication, Paris, Stock.

MONTAGNER, H. 1988 L'attachement, les débuts de la tendresse, Paris, Odile Jacob.

MONTAGNER, H. 1993 L'enfant acteur de son développement, Paris, Stock.

MONTAGNER, H. 1995 « Attachement, tendresses et vicissitudes. Les compétences-socles de l'enfant », in M. Gabel, S. Lebovici, Ph. Mazet et al., Maltraitance : maintien du lien ?, 65-98, Paris, Fleurus.

MONTAGNER, H. 1996 En finir avec l'échec à l'école, Paris, Bayard.

MONTAGNER, H. 1997 « Les rythmes de l'enfant », in Petite enfance, éveil aux savoirs, 349-358, Paris, Documentation Française, Ministère du Travail et des Affaires Sociales.

MONTAGNER, H., VAN LEUWEN, F., BLANC, I., GAUFFIER, G., MAURY, M., VISIER, J.P. 1989 « Une nouvelle approche méthodologique du développement de l'enfant et de ses systèmes interactifs au cours de la période postnatale », in S. Lebovici, P. Mazet et J.P. Visier (eds) «L'évaluation des interactions précoces entre le bébé et ses partenaires ", 99-130, Paris, Ed. Eshel.

MONTAGNER, H., MAGNUSSON, M., CASAGRANDE, C, RESTOIN, A., BEL, J.P, NGUYEN HOANG, P., RUIZ, V., DELCOURT, S., GAUFFIER, G., EPOULET, B. 1990 « Une nouvelle méthode pour l'étude des organisateurs de comportements et des systèmes d'interaction du jeune enfant. Les premières données ", Psychiatrie de l’Enfant, 33, 391-456.

MONTAGNER, H., RUIZ, V., RAMEL, N., RESTOIN, A., MERTZIANIDOU, V., GAUFFIER, G. 1993, « Les capacités interactives d'enfants de 3 à 7 mois avec un enfant du même âge ", Psychiatrie de l'Enfant, 36, 489-536.

MONTAGNER, H., GAUFFIER, G., EPOULET, B., RESTOIN, A., GOULEVITCH, R., TAULE, M., WIAUX, B. 1993 « Alternative child care in France. Advances in the study of motor, interactive and social behaviors of young children in settings allowing them to move freely in a group of peers ", Pediatrics, 91, 253-263.

MONTAGNER, H., EPOULET, B., GAUFFIER, G., GOULEVITCH, R., RAMEL, N., WIAUX, B., TAULE, M. 1994 The earliness and complexity of the interaction skills and social behaviors of the child with its peers, in R.A. Gardner, B.T. Gardner, B. Chiarelli and F.X. Plooij (eds) The ethological roots of culture, Nato Asi Series, Series D: Behavioral and Social Sciences, 78, 315-355, Dordrecht (Holland), Kluwer Academic Publ.

PAGES, S. 1996 « Influence des variables différentielles sur les réponses motrices des bébés nés par voie naturelle et des bébés nés par césarienne à des stimulations auditives ». Thèse de Doctorat en Sciences de la Vie et de la Santé, Université de Montpellier I.

SPITZ, R.A. 1945 « Hospitalism: An inquiry into the psychiatric conditions in early childhood, » Psychoanal. Study Child, 1, 53-74.

SPITZ, R.A. 1946 « Anaclitic depression », Psychoanal. Study Child, 2, 313-342.

SPITZ, R.A. 1968 De la naissance à la parole, Paris, P.U.F.

SPITZ, R..A. and Wolff, K.M. 1946 « The smiling response: A contribution to the ontogenesis of social response », Genetics Psychology Monographs, 34, 57-125. 
STERN, D. 1982 « Some interactive functions of rhythm changes between mother and infant », in M. Davis (ed.) Interaction rhythms. Periodicity in communication behavior, 101-117, New York, Human Sciences Press.

STERN, D. 1985 « Affect attunement: Mechanisms and clinical implications », in J.D. Call, E. Galenson and R.L. Tyson (eds) Frontiers of infant psychiatry, 2, New York, Basic Books.

STERN, D. 1985 The interpersonal world of the infant, New York, Basic Books.

TRONICK, E. Als, H., Brazelton, T.B. 1977 « Mutuality in mother-infant interaction », /. of Communication Information Processing, 27, 74-79.

TRONICK, E., Als, H., Adamson, L., Wise, S., Brazelton, T.B. 1978 « The infant's response to entrapment between contradictory messages in face-to-face interaction », /. Child Psychiatry, 17, 1-13.

TRONICK, E., ALS, H., Brazelton, T.B. 1980 » Monadic phases: A structural descriptive analysis of infant-mother face-to-face interaction ", Merril-Palmer Quaterly, 26, 3-23.

TRONICK, E. COHN, J. SHEA, E. 1984 « The transfer of affect between mothers and infant ", in T.B. Brazelton and M.W. Yogman (eds) Affective Development in Infancy, Norwood (N.J.), Ablex.

\section{AUTEUR}

\section{HUBERT MONTAGNER}

Département de Pédopsychiatrie, Hôpital Sainte-Marguerite, Marseille, Université d'AixMarseille II 